\title{
Investigation of optical coherence micro- elastography as a method to visualize micro-architecture in human axillary lymph nodes
}

Kelsey M. Kennedy ${ }^{1+}$, Lixin Chin ${ }^{1,2^{*}+}$, Philip Wijesinghe ${ }^{1,2}$, Robert A. McLaughlin ${ }^{1,3}$, Bruce Latham ${ }^{4}$, David D. Sampson ${ }^{1,5}$, Christobel M. Saunders ${ }^{6,7}$ and Brendan F. Kennedy ${ }^{1,2}$

\begin{abstract}
Background: Evaluation of lymph node involvement is an important factor in detecting metastasis and deciding whether to perform axillary lymph node dissection (ALND) in breast cancer surgery. As ALND is associated with potentially severe long term morbidity, the accuracy of lymph node assessment is imperative in avoiding unnecessary ALND. The mechanical properties of malignant lymph nodes are often distinct from those of normal nodes. A method to image the micro-scale mechanical properties of lymph nodes could, thus, provide diagnostic information to aid in the assessment of lymph node involvement in metastatic cancer. In this study, we scan axillary lymph nodes, freshly excised from breast cancer patients, with optical coherence micro-elastography (OCME), a method of imaging micro-scale mechanical strain, to assess its potential for the intraoperative assessment of lymph node involvement.
\end{abstract}

Methods: Twenty-six fresh, unstained lymph nodes were imaged from 15 patients undergoing mastectomy or breast-conserving surgery with axillary clearance. Lymph node specimens were bisected to allow imaging of the internal face of each node. Co-located OCME and optical coherence tomography (OCT) scans were taken of each sample, and the results compared to standard post-operative hematoxylin-and-eosin-stained histology.

Results: The optical backscattering signal provided by OCT alone may not provide reliable differentiation by inspection between benign and malignant lymphoid tissue. Alternatively, OCME highlights local changes in tissue strain that correspond to malignancy and are distinct from strain patterns in benign lymphoid tissue. The mechanical contrast provided by OCME complements the optical contrast provided by OCT and aids in the differentiation of malignant tumor from uninvolved lymphoid tissue.

Conclusion: The combination of OCME and OCT images represents a promising method for the identification of malignant lymphoid tissue. This method shows potential to provide intraoperative assessment of lymph node involvement, thus, preventing unnecessary removal of uninvolved tissues and improving patient outcomes.

Keywords: Breast cancer, Lymph node, Optical coherence tomography, Elastography, Mechanical properties, Intraoperative

\footnotetext{
* Correspondence: lixin.chin@research.uwa.edu.au

${ }^{\dagger}$ Equal contributors

${ }^{1}$ Optical+Biomedical Engineering Laboratory, School of Electrical, Electronic \&

Computer Engineering, The University of Western Australia, 35 Stirling

Highway, Perth, WA 6009, Australia

${ }^{2}$ BRITElab, Harry Perkins Institute of Medical Research, QEll Medical Centre, 6

Verdun St, Nedlands, Perth, WA 6009, Australia

Full list of author information is available at the end of the article
} 


\section{Background}

The presence of lymph node metastasis is one of the primary prognostic indicators for patients with earlystage breast cancer [1-3]. During surgery, the sentinel lymph node (SLN) (the first-draining node) is often excised and assessed. If the SLN is found to be involved, axillary lymph node dissection (ALND) is often performed to determine if the disease is present in the lymphatic system and to prevent its further spread [3]. However, ALND is associated with potentially significant long term morbidity, such as seroma formation, altered sensation in the upper limb, and lymphedema [4]. The accuracy of SLN assessment is, thus, imperative in avoiding unnecessary ALND. Current pre- and intraoperative SLN assessment techniques include macroscopic examination, pathology, and medical imaging [5]. Macroscopic assessment is subjective; for example, when performing visual inspection and palpation alone, inflammation in nodes may be mistaken for malignancy [6]. Intraoperative pathology techniques, including frozen section and imprint cytology, provide high-resolution, highly specific assessment of lymph node morphology, but are often expensive, time-consuming (20-40 min), and can sample only a small proportion of the lymph node within an intraoperative timeframe [7]. This can result in low diagnostic accuracy: the sensitivities of frozen section and imprint cytology have been reported to be in the range $53-74 \%$, with corresponding specificities of 98-100\% [8, 9]. Medical imaging, including magnetic resonance imaging (MRI) [10], ultrasound (US) [11], and positron emission tomography/X-ray computed tomography (PET-CT) [12], have also been proposed for preoperative staging of the axilla. These techniques rely predominantly on assessment of the size and shape of the nodes, as they do not typically have the resolution required to visualize micro-scale lymph node morphology, limiting their potential for SLN assessment. For example, these medical imaging techniques can have difficulty in distinguishing metastatic and reactive nodes [11].

New techniques for rapid, accurate, intraoperative assessment of the SLN have the potential to improve surgical decision-making, ultimately leading to improved patient outcomes. Methods such as high-frequency ultrasound (HF-US) [13] and photoacoustic tomography (PAT) [14] have been investigated, reporting spatial resolutions of $\sim 100 \mu \mathrm{m}$ (HF-US) or $\sim 50 \mu \mathrm{m}$ (PAT); additionally, PAT has been used in conjunction with external contrast agents. To obtain improved spatial resolution without the need for labelling agents, optical modalities, such as optical coherence tomography (OCT), often described as the optical analog to ultrasonography, have been proposed [15-22]. The detection of backscattered light, rather than sound, provides OCT with a spatial resolution of 1$20 \mu \mathrm{m}$, but only to a depth of $1-2 \mathrm{~mm}$ in turbid tissue.
OCT has the potential to rapidly scan entire ex vivo lymph nodes intraoperatively, improving upon the sampling issues of intraoperative histopathology techniques. However, it is unclear if lymph node metastases can be adequately delineated based on endogenous optical scattering contrast alone [21, 22].

It has previously been established that the mechanical properties of malignant lymph nodes are often distinct to those of normal nodes [23, 24]. Mapping the microscale mechanical properties of lymph nodes could, thus, provide additional diagnostic information to complement that provided by OCT. Over the past 25 years, various imaging techniques, collectively termed elastography, have emerged to map the mechanical properties of tissues. Elastography uses an imaging modality, most commonly US [25] or MRI [26], to measure tissue deformation in response to a mechanical load. Tissue mechanical properties are then estimated using a mechanical model, and mapped onto an image, known as an elastogram. Several studies have investigated the use of US elastography to assess axillary lymph nodes [27, 28]. However, the spatial resolution of elastography is ultimately limited by the underlying imaging modality, which, using US or MRI, may be insufficient to detect lymph node metastasis.

Optical coherence elastography (OCE) [29-32] uses OCT as the underlying modality and, thus, has a higher spatial resolution than US- or MRI-based elastography [30]. Our group has recently demonstrated a variant of OCE, dubbed optical coherence micro-elastography (OCME), which has a lateral resolution matched to that of OCT over en face fields of view up to $\sim 20 \times 20 \mathrm{~mm}$ [33]. In OCME, a uniform compressive load is applied to the specimen, and 3-D elastograms are generated of the resulting mechanical strain, which is inversely proportional to the tissue stiffness. We have shown that this technique can provide contrast between malignant and normal tissue in fresh, excised human breast specimens [33, 34].

Here, we utilized OCME to scan axillary lymph nodes, freshly excised from human breast cancer patients, to investigate its potential for the intraoperative assessment of lymph nodes. We imaged 26 specimens from 15 patients undergoing a mastectomy or breast-conserving surgery with axillary clearance and present co-located histology, OCT images, and elastograms of selected representative cases. The results show that OCME provides contrast that is additional and complementary to that provided by OCT and demonstrate the potential of OCME for intraoperative SLN assessment.

\section{Methods}

Imaging system

A portable OCE system, described in detail previously $[33,34]$, was used in this study. The system utilizes an 
835-nm, spectrometer-based OCT system, with axial/ lateral resolutions of $8 \mu \mathrm{m} / 11 \mu \mathrm{m}$ (in air), respectively. 3 -D datasets were acquired in $\sim 17 \mathrm{~min}$, with dimensions $(x \times y \times z)$ up to $10 \times 10 \times 1.5 \mathrm{~mm}$, comprising 1000 line scans (A-scans) in each 2-D image (B-scan) and 10,000 B-scans in each volume (C-scan). An annular piezoelectric transducer (Piezomechanik, Germany) with a glass window fixed to the surface was used to enable imaging and mechanical loading from the same side of the sample [33,34]. Figure 1 shows a schematic diagram of this imaging system.

\section{Imaging protocol}

To ensure even contact with the window, the specimen was preloaded prior to imaging by applying 10-20\% compression. During imaging, the transducer displaced the tissue surface by up to a maximum of $\sim 2 \mu \mathrm{m}$, at a frequency of $5 \mathrm{~Hz}$, and was synchronized with the OCT acquisition such that consecutive B-scans were acquired in the unloaded then loaded state. The local axial tissue displacement was proportional to the phase difference between corresponding A-scans in consecutive B-scans, as measured using phase-sensitive OCT [35]. Five pairs of B-scans were recorded and averaged at each $y$-position to improve the precision of the measured displacement [33]. A phase-unwrapping algorithm, described previously, was used to improve the dynamic range of measurable displacements [33]. Elastograms were generated from the displacement maps by calculating the local

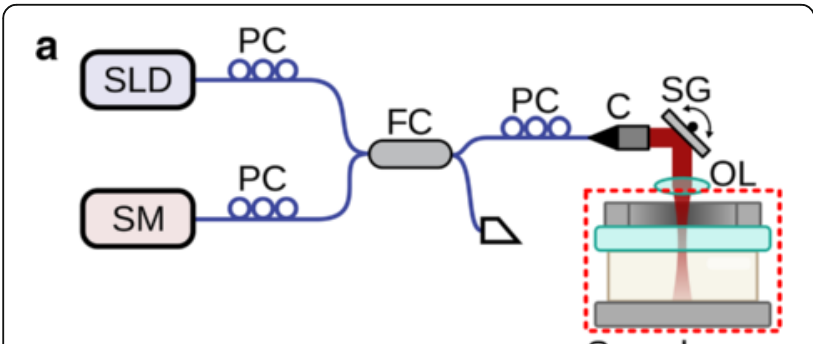

Sample arm

b

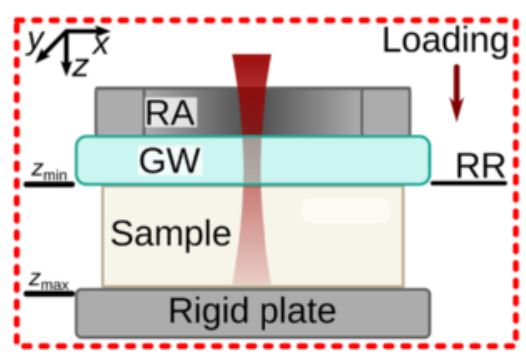

Fig. 1 a Schematic of the imaging system used in this study. Collimator (C), 50/50 fiber coupler ( $\mathrm{FC}$ ), objective lens (OL), polarization controller $(\mathrm{PC})$, scanning galvanometer $(\mathrm{SG})$, superluminescent diode light source (SLD), spectrometer (SM). b Schematic (side view) of the sample stage, corresponding to the region within the red box in (a). Glass window (GW), piezoelectric ring actuator (RA), reference reflector (RR) strain (change in displacement assessed over an axial depth range of $100 \mu \mathrm{m}$, which defined the elastogram axial resolution) throughout the sample using a weighted least-squares linear regression algorithm [33]. The lateral $(x, y)$ resolution of the elastograms matched that of the OCT system $(11 \mu \mathrm{m})$. All datasets were processed using custom software implemented in Matlab (Mathworks, USA, v2012b).

OCT is effective in identifying adipose, which exhibits optical scattering patterns distinct from those of other tissues. However, the low OCT signal within adipose cells introduces significant noise into the elastograms. Adipose, and other areas of low OCT signal, were manually segmented from the elastograms using image processing software (GNU Image Manipulation Program, v2.8.14), and the remaining OCME data (elastogram) overlaid onto the OCT image [34]. Elastograms are presented on a linear false-color scale in millistrain $(\mathrm{m} \varepsilon)$, i.e., length change per unit length $\times 10^{-3}$, and OCT images are presented on a logarithmic decibel $(\mathrm{dB})$ grayscale. As the amount of contrast provided by OCME is dependent on the sample structure and applied load [34] (see Discussion), the color-scale of the presented elastograms was chosen to maximize the available image contrast. The presented en face OCT images and elastograms are taken from within $300 \mu \mathrm{m}$ of the sample surface, chosen as the en face images that best matched the corresponding histology.

\section{Tissue preparation}

Informed consent was obtained from patients and the study approved by the Human Ethics Committee of Sir Charles Gairdner Hospital, Perth, Western Australia. Twenty-six specimens were imaged from 15 patients undergoing a mastectomy or breast-conserving surgery with axillary clearance. Samples were bisected to allow imaging of the internal face of each node and kept hydrated in saline until imaging, which occurred within 1$2 \mathrm{~h}$ of excision. After imaging, specimens were fixed in $10 \%$ neutral buffered formalin, embedded in paraffin, sectioned and stained with hematoxylin and eosin (H\&E), following standard histopathology protocols. The H\&E-stained sections were digitally micrographed (ScanScope, Leica Biosystems, Nussloch, Germany) and manually co-registered with the corresponding en face elastograms and en face OCT images. Interpretation of histology was performed by an experienced pathologist (B. Latham).

\section{Results}

Figure 2 shows an example of a normal, benign lymph node. The histology (Fig. 2a) shows that the node has a fibrous capsule $(\mathrm{C})$ separating the lymphoid tissue from surrounding adipose (A). Several follicles (F) comprising 


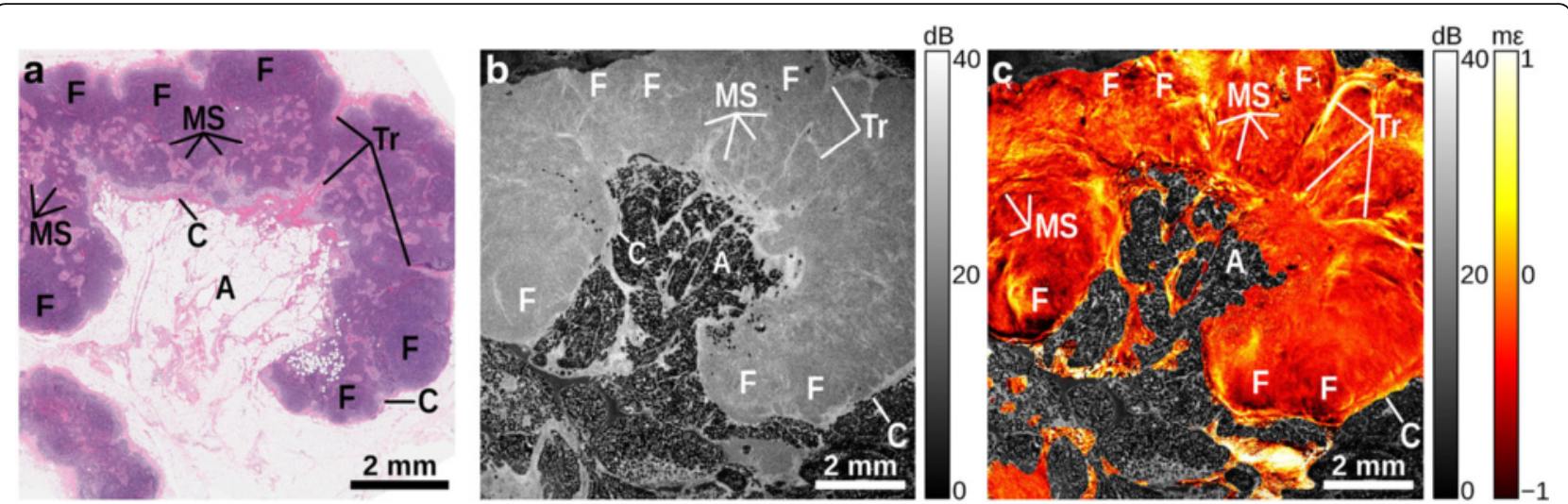

Fig. 2 Benign lymph node sample. a H\&E-stained histology, (b) en face OCT image, and (c) elastogram overlaid on OCT. Adipose (A), capsule (C), follicle (F), medullary sinuses (MS), trabeculae (Tr)

sheets of lymphocytes are observed in the cortex of the node. Below the cortex, the medulla comprises medullary cords separated by medullary sinuses (MS). Fibrous trabeculae (Tr) are also visible. In the OCT image (Fig. 2b), the fibrous capsule is distinguished by higher backscattering, and the surrounding adipose provides a distinctive honeycomb structure. Thin strands of high backscattering, corresponding to collagen fibers in the trabeculae and medullary sinuses, are present throughout the node. In the elastogram (Fig. 2c), the capsule is identified by an abrupt change in strain. The elastogram provides additional contrast of trabeculae compared to the OCT. The follicles present as oval-shaped regions that are separated by changes in strain caused by surrounding trabeculae and sinuses. Smaller, irregularly shaped local areas of greater negative (compressive) strain in the medulla correspond to medullary sinuses.

Figure 3 shows a lymph node containing a $\sim 3 \mathrm{~mm}$ diameter region of metastatic tumor $(\mathrm{T})$. The remainder of the sample is normal lymphoid tissue and contains several follicles $(\mathrm{F})$ with relatively large germinal centers. Fibrous trabeculae (Tr) are also observed running through the normal portion of the node. The OCT image (Fig. 3b) highlights areas of collagen, including the trabeculae, and some follicles are also distinguishable. The OCT image does not, however, provide contrast between the normal and malignant regions. On the other hand, the tumor is readily differentiated from normal tissue in the elastogram (Fig. 3c). Strain heterogeneity within the malignant region is attributed to groups of tumor cells separated by fibrous bands. Within the normal region of the lymph node, pockets of high negative strain correspond to germinal centers within follicles.

Figure 4 shows a lymph node containing a $\sim 3 \times 1 \mathrm{~mm}$ region of metastatic tumor $(\mathrm{T})$ classified as pleomorphic lobular carcinoma. The top left of the node contains mainly sheets of tumor cells, and these become gradually more interspersed with lymphocytes and macrophages toward the right of the sample. The far right of the sample is normal lymphoid tissue and contains follicles (F). The central region consists of a band of stroma, containing several vessels $(\mathrm{V})$, surrounded by adipose (A). The OCT image (Fig. 4b) again exhibits higher backscattering in the areas of dense stroma, including the capsule (C), but presents an otherwise homogeneous signal. The elastogram (Fig. 4c) reveals a region of high negative strain at the far left corresponding to a region of solid tumor cells delineated by stroma. The elastogram is also highly heterogeneous in the central region of stroma, particularly around vessels. The remainder of the region of solid tumor cells presents as mostly homogeneous strain. The far right of the sample, corresponding to normal lymph node, contains small, local areas of negative strain, more similar to the heterogeneity seen in areas of normal lymph node in Figs. 2 and 3.

Figure 5 shows a region of a lymph node in which normal tissue has been largely replaced by malignancy. The histology reveals an area of fibrotic stroma (S) toward the center of the sample, with an area of necrosis $(\mathrm{N})$ present, surrounded by tumor interspersed with stroma (T). In the OCT image (Fig. 5b), the capsule (C), as well as the region of fibrosis, can be identified as regions of higher backscattering. The elastogram (Fig. 5c) provides much higher contrast between the capsule and the stroma than OCT. The region of fibrosis presents as homogeneous strain, indicating its mechanical uniformity. In areas where tumor cells are interspersed with desmoplastic stroma, heterogeneous strain is present.

\section{Discussion}

In this study, we have presented representative elastograms, with corresponding OCT and histology, selected from 26 fresh, unstained specimens. In benign and involved lymph 

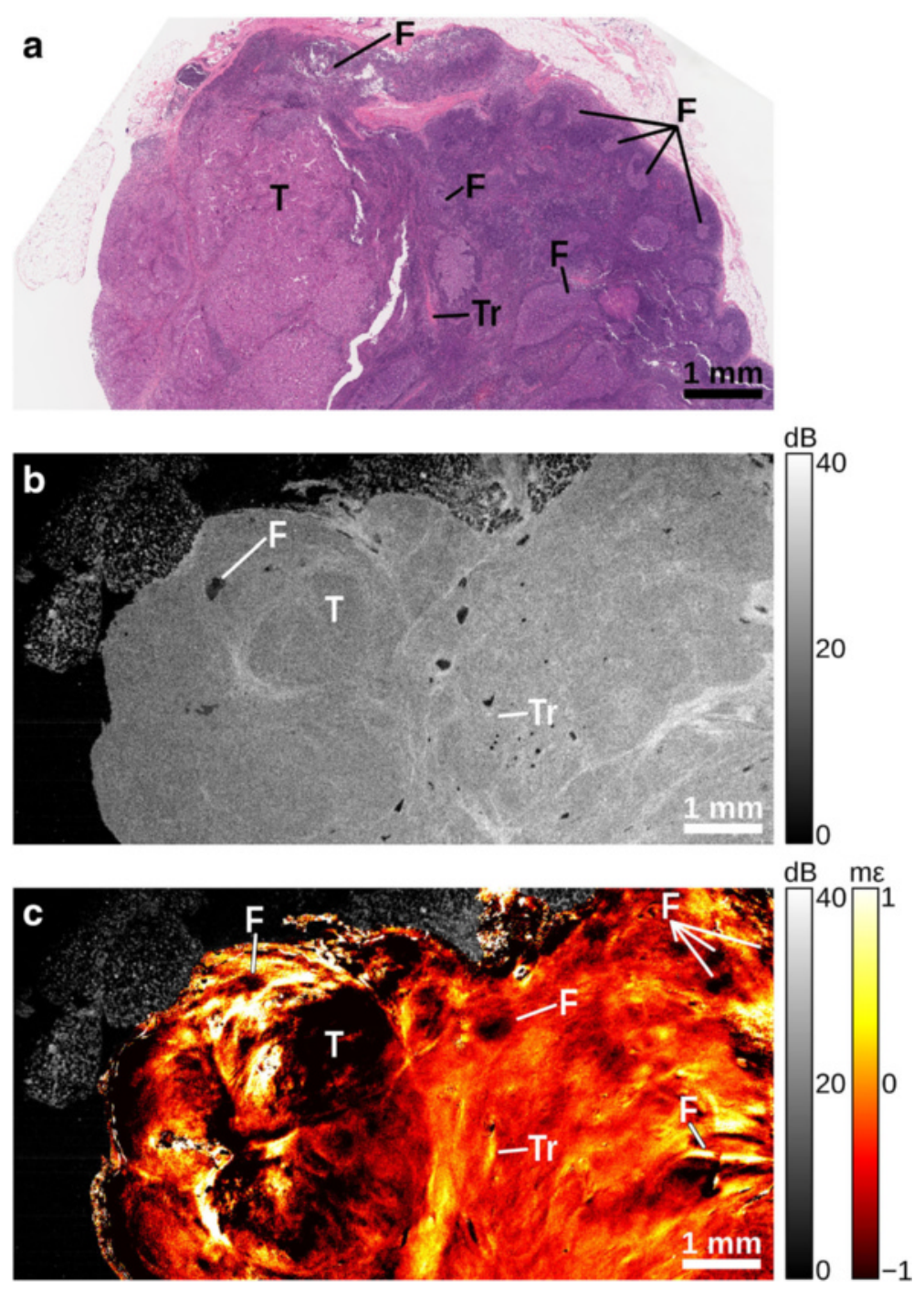

Fig. 3 Lymph node containing metastasis. a H\&E-stained histology, (b) en face OCT image, and (c) elastogram overlaid on OCT. Follicle (F), tumor (T), trabeculae $(\mathrm{Tr})$

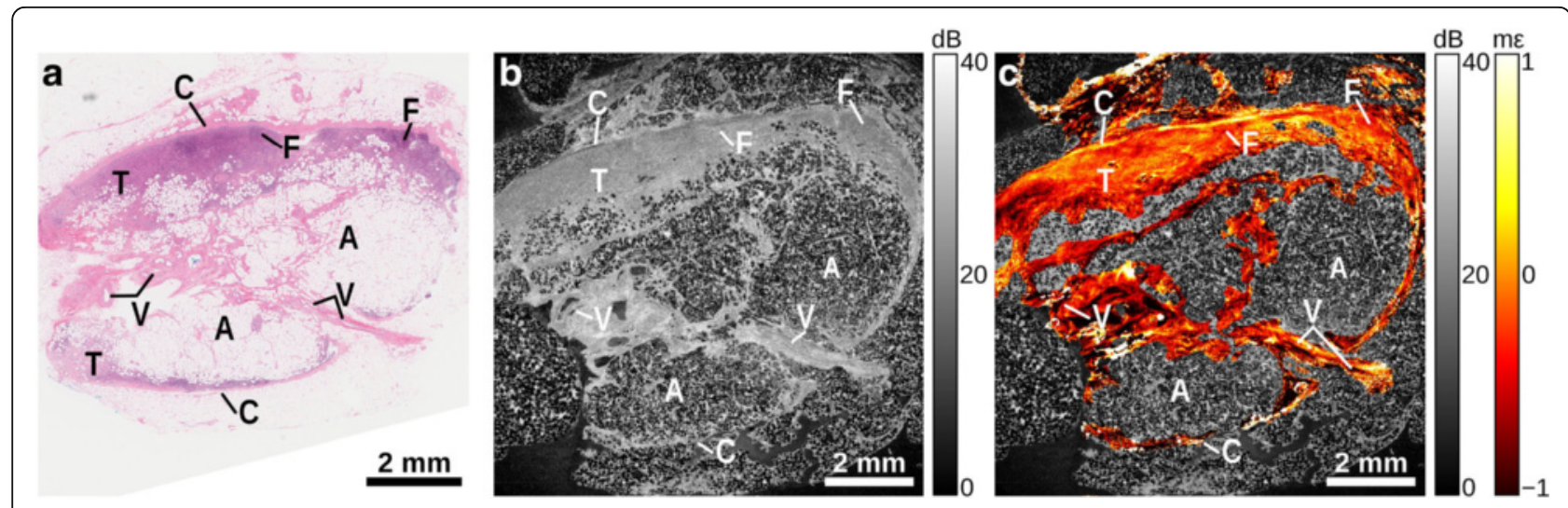

Fig. 4 Lymph node containing pleomorphic lobular carcinoma. a H\&E-stained histology, (b) en face OCT image, and (c) elastogram overlaid on OCT. Adipose (A), capsule (C), follicle (F), tumor (T), vessel (V) 


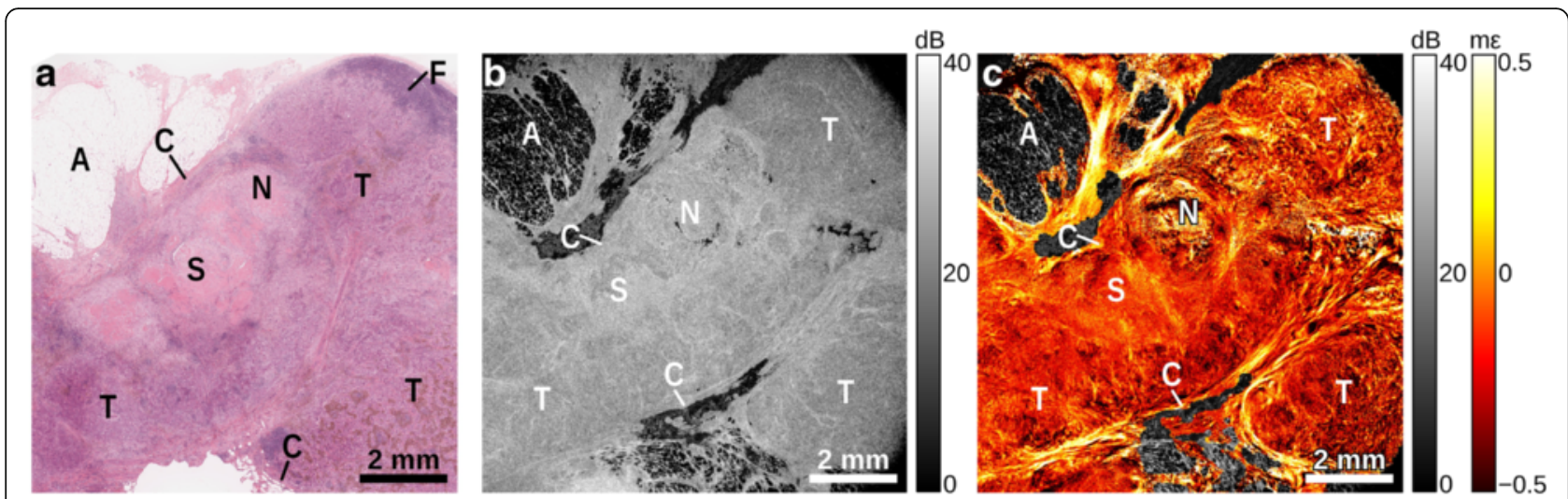

Fig. 5 Lymph node largely replaced by metastasis. a H\&E-stained histology, (b) en face OCT image, and (c) elastogram overlaid on OCT. Adipose $(A)$, capsule $(C)$, necrosis $(N)$, stroma $(S)$, tumor $(T)$

nodes, we observed features such as adipose, capsule, follicles, and trabeculae. As with our previous OCME studies on human breast tissue [33, 34], the results show that OCME provides additional contrast that is complementary to that provided by OCT alone. This complementary information is presented using image fusion to combine the en face elastograms with the OCT images, in a similar manner to that employed by existing medical imaging techniques, such as MRI and PET-CT [36]. The contrast observed in the elastograms consists of both negative (compressive) strain, as expected, and also positive (tensile or compressive in the direction opposed to the applied force) strain. This accentuates feature contrast, and is a reflection of the structural heterogeneity of the tissue [34].

In the OCT images (Part b, Figs. 2, 3, 4 and 5), adipose is readily distinguished by its distinct 'honeycomb' appearance. The capsule surrounding the lymph node can be seen as a band of higher backscatter in OCT, and as abrupt changes in strain in OCME. Follicles make up much of the structure of healthy lymph nodes (Fig. 2), with the trabeculae between the follicles visible as bands of higher backscattering (OCT, Fig. 2b), and discontinuities in the strain pattern (OCME, Fig. 2c). Regions of tumor typically appear in the OCT images as areas of homogeneous signal, with few distinguishing features (Part b, Figs. 3, 4 and 5). The same regions can often be distinguished in the elastograms (Part c, Figs. 3, 4 and 5), exhibiting strain patterns distinct from that of uninvolved follicles (Fig. 2c). Consistent with our previous studies in breast tissue, regions of solid tumor cells result in homogeneous strain patterns; whereas, regions containing tumor mixed with desmoplastic stroma result in heterogeneous strain [34]. On the cellular level, tumor cells are often softer than the collagen-rich stroma [37, 38], which is likely the cause of the heterogeneous strain patterns observed in these mixed regions. Although the appearance of tumor varies notably from specimen to specimen, in all the cases we examined the strain patterns can be linked to the underlying micro-scale tissue constituents and architecture and, in most cases, the mechanical contrast in regions of malignancy improves upon that provided by OCT alone. This is most noticeable in Fig. 5, where the fine structure seen in the OCME of the tumor on the right of the image is absent from the OCT images. Further study is required to ascertain the extent to which these visual features are consistent over the larger population. However, we have found that the mechanical contrast provided by OCME complements the optical contrast provided by OCT, and aids in the distinction of malignant from benign lymph node tissue.

Previous studies in assessing lymph node involvement using OCT imaging alone have reported sensitivity/ specificities of $92 \% / 83 \%$ by Grieve et al. [21], and $59 \% / 81 \%$ by Nolan et al. [22]. Grieve et al.'s results demonstrate the benefits of using high resolution OCT ( $\sim 1 \mu \mathrm{m}$, approaching the $\sim 0.5 \mu \mathrm{m}$ of typically histology). However, this high resolution is achieved at the expense of a low depth of field (DOF, $\sim 10 \mu \mathrm{m}$, using a white light source and a water immersion lens with a numerical aperture of 0.3) [21], compared to the DOF of $\sim 0.5 \mathrm{~mm}$ of standard OCT (near-infrared light, lens numerical aperture of $\sim 0.03-0.04$ ). Low DOF typically implies that either the sample or the imaging lens must be physically moved in order to acquire in-focus images from multiple depths, slowing acquisition of 3-D volumes. Nolan et al. used an OCT system with comparable resolution to that used in this study $(\sim 10 \mu \mathrm{m})$ [22], and their results support our findings that OCT alone may not be sufficient in reliably detecting lymph node metastases. Nolan et al. additionally examined multiple slices through the volume in order to arrive at a diagnosis; whereas, our study analyzed a single OCT/ OCME en face image for each sample, chosen from the depth that best matched the histology section. Full 3-D examination may potentially allow for improved diagnosis within a short time frame, and further study is 
required to assess the information available from the full 3-D OCME dataset.

The images presented in this study were obtained from within $300 \mu \mathrm{m}$ of the bisected surface of the lymph nodes. The depth penetration of our OCT system in human lymphoid tissue was observed to be approximately $500-700 \mu \mathrm{m}$; however, the image quality of both OCT and OCME notably degrades due to attenuation of the light beam by scattering as we approach this maximum depth. Bisecting the nodes, as in this study, allows scanning of two internal faces and effectively doubles the depth information obtained. In future intraoperative deployment, both the internal and external faces of bisected nodes could be scanned, which we estimate would allow sampling of $>50 \%$ of the volume of most lymph nodes, much greater than that achievable using intraoperative histopathology [39]. The percentage of the lymph node that should be sampled for OCME to accurately judge involvement status will form part of a future study aimed at assessing the sensitivity and specificity of the technique.

Measurement of local strain in OCME is coupled to the underlying sample structure and elasticity, and to the applied load [30, 33, 34]. Related OCE techniques have been demonstrated that directly estimate elasticity based on measurement of the propagation velocity of elastic waves [30-32, 40-42]. However, these techniques have yet to overcome the challenges necessary to achieve spatial resolutions comparable with compression OCE techniques, such as OCME [30-32]. We have recently shown an adaptation of OCME that determines elasticity at the surface of tissue, using a compliant silicone layer with known mechanical properties to account for applied load [43]. However, the ability to characterize the elasticity distribution at depth in heterogeneous tissues is still to be fully investigated. Another possible solution is to solve for the elasticity distribution using an iterative computational method [44]. In addition, semi-quantitative assessment may be possible using a visual grading scale, relating features seen in OCT and OCME with the presence and type/grade of tumor.

The results of this study demonstrate that OCME can visualize multiple salient features of the microarchitecture of malignant and benign nodes, providing justification for a larger study, incorporating more specimens and patients, in order to determine the diagnostic accuracy of the technique (sensitivity and specificity). Additional study is also required to determine the minimum metastasis size that can be reliably identified with OCME. We hypothesize that this size will be dependent on the differences between the mechanical properties of the particular tumor type and normal lymphoid tissue. This lower bound is likely to be larger than the smallest metastasis detectable using histology, as the lateral resolution of OCME $(\sim 10 \mu \mathrm{m})$ is much coarser than that of the optical microscopes used for histology $(<0.5 \mu \mathrm{m})$. However, we have recently demonstrated a variant of OCME with a lateral resolution of $2 \mu \mathrm{m}$ [45] that may be able to detect micrometastases of a similar size to those detectable with histology, and further improvements may be possible by using an OCT system with even higher resolution $(<1 \mu \mathrm{m})$ [21]. Future studies will also aim to further develop OCME toward providing results within intraoperative timeframes, which will require significant increases to acquisition and processing speeds. The current study acquired 3-D volumes in $~ 17 \mathrm{~min}$; however, we have previously demonstrated a method of acquiring OCME volumes in $\sim 5 \mathrm{~s}$ [46], which could be applied to scanning lymph nodes. Similarly, using the current software, each dataset required approximately $1 \mathrm{~h}$ to process on a standard PC. We have recently shown the use of custom software running on a graphics processing unit to decrease this time to under $50 \mathrm{~s}$ for datasets of the size recorded in this study [47]; however, further research is required to ensure the image quality matches that of the results shown here.

\section{Conclusion}

OCME represents a new method for the identification of malignant tissue in lymph nodes, with the potential to be used intraoperatively. In this initial study, we have demonstrated the correspondence between elastograms and co-registered histology, as well as the complementary information provided by OCT and OCME. These results show the potential for future development of OCME as an intraoperative tool for assessment of lymph node involvement.

\section{Abbreviations}

ALND: Axillary lymph node dissection; H\&E: Hematoxylin and eosin; MRI: Magnetic resonance imaging; OCE: Optical coherence elastography; OCME: Optical coherence micro-elastography; OCT: Optical coherence tomography; PAT: Photoacoustic tomography; PET-CT: Positron emission tomography/X-ray computed tomography; SLN: Sentinel lymph node; US: Ultrasound

\section{Acknowledgements \\ The authors acknowledge the facilities, and the scientific and technical assistance of the Australian Microscopy and Microanalysis Research Facility at the Centre for Microscopy, Characterization and Analysis, The University of Western Australia, a facility funded by the University, State and Commonwealth Governments. We acknowledge the support of Maxine Ronald, Jocelyn Lippey, Derek Teh and Wes Allen in acquiring, processing and interpreting the data.}

\section{Funding}

This project is supported with funding from the Australian Research Council; and Cancer Council WA, which allowed us to develop the technology; Department of Health of Western Australia, National Breast Cancer Foundation (Australia); and the National Health and Medical Research Council, Australia, which allowed us to design and implement the clinical protocol, and to perform the clinical measurements. 


\section{Availability of data and materials}

The datasets analyzed during this study are available from the authors on reasonable request.

\section{Authors' contributions}

KMK and BFK designed and performed the experiments and analyzed the data. LC developed the signal processing chain. PW and RAM assisted with the imaging experiments. BL performed tissue dissection and interpreted histology. RAM, CMS and BFK coordinated clinical imaging. CMS performed surgeries and facilitated access to tissue. DDS and BFK conceived, directed and guided the work. KMK, LC and BFK wrote the manuscript, which was reviewed and revised by all authors. All authors read and approved the final manuscript.

\section{Competing interests}

The authors are currently in negotiations to explore commercialization of optical coherence micro-elastography.

\section{Consent for publication}

Written informed consent for publication of their clinical data was obtained from all patients. A copy of the consent form is available for review by the Editor of this journal.

\section{Ethics approval and consent to participate}

Informed consent was obtained from patients and the study approved by the Human Ethics Committee of Sir Charles Gairdner Hospital, Perth, Western Australia.

\section{Author details}

'Optical+Biomedical Engineering Laboratory, School of Electrical, Electronic \& Computer Engineering, The University of Western Australia, 35 Stirling Highway, Perth, WA 6009, Australia. ${ }^{2}$ BRITElab, Harry Perkins Institute of Medical Research, QEll Medical Centre, 6 Verdun St, Nedlands, Perth, WA 6009, Australia. ${ }^{3}$ Australian Research Council Centre of Excellence for Nanoscale BioPhotonics, School of Medicine, Faculty of Health Sciences, University of Adelaide, Adelaide, SA 5005, Australia. ${ }^{4}$ PathWest, Fiona Stanley Hospital, Robin Warren Drive, Murdoch, WA 6150, Australia. ${ }^{5}$ Centre for Microscopy, Characterisation \& Analysis, The University of Western Australia, 35 Stirling Highway, Perth, WA 6009, Australia. ${ }^{6}$ School of Surgery, The University of Western Australia, 35 Stirling Highway, Perth, WA 6009, Australia. 'Breast Clinic, Royal Perth Hospital, 197 Wellington Street, Perth, WA 6000, Australia.

\section{Received: 6 June 2016 Accepted: 27 October 2016} Published online: 09 November 2016

\section{References}

1. Overgaard J. Management of the axilla in breast cancer: Implication for diagnosis, prognosis, treatment, and morbidity. Acta Oncol. 2000;39(3):259-60.

2. Weigelt $B$, Peterse $J L$, van't Veer $L J$. Breast cancer metastasis: Markers and models. Nat Rev Cancer. 2005;5(8):591-602.

3. Lyman GH, Temin S, Edge SB, Newman LA, Turner RR, Weaver DL, Benson III AB, Bosserman LD, Burstein HJ, Cody III H, et al. Sentinel lymph node biopsy for patients with early-stage breast cancer: American Society of Clinical Oncology clinical practice guideline update. J Clin Oncol. 2014;32(13):1365-83.

4. Sakorafas GH, Peros G, Cataliotti L. Sequelae following axillary lymph node dissection for breast cancer. Expert Rev Anticancer Ther. 2006;6(11):1629-38.

5. Valente SA, Levine GM, Silverstein MJ, Rayhanabad JA, Weng-Grumley JG, Ji L, Holmes DR, Sposto R, Sener SF. Accuracy of predicting axillary lymph node positivity by physical examination, mammography, ultrasonography, and magnetic resonance imaging. Ann Surg Oncol. 2012;19(6):1825-30.

6. Fujii T, Yanagita Y, Kinoshita T, Fujisawa T, Hirakata T, Yamaki S, Matsumoto A, Uchida N, lijima M, Kuwano H. Accuracy of intraoperative macroscopic diagnosis of sentinel node metastases in breast cancer: Is accurate prediction possible? Tumori. 2011;97(1):62-5.

7. Layfield DM, Agrawal A, Roche H, Cutress Rl. Intraoperative assessment of sentinel lymph nodes in breast cancer. Br J Surg. 2011;98(1):4-17.

8. Tanis PJ, Boom RPA, Koops HS, Faneyte IF, Peterse JL, Nieweg OE, Rutgers EJT, Tiebosch ATMG, Kroon BBR. Frozen section investigation of the sentinel node in malignant melanoma and breast cancer. Ann Surg Oncol. 2001;8(3):222-6.
9. Creager AJ, Geisinger KR, Shiver SA, Perrier ND, Shen P, Shaw JA, Young PR, Levine EA. Intraoperative evaluation of sentinel lymph nodes for metastatic breast carcinoma by imprint cytology. Mod Pathol. 2002;15(11):1140-7.

10. Fischer U, Zachariae O, Baum F, Heyden D, Funke M, Liersch T. The influence of preoperative MRI of the breasts on recurrence rate in patients with breast cancer. Eur Radiol. 2004;14(10):1725-31.

11. Diepstraten SCE, Sever AR, Buckens CFM, Veldhuis WB, Dalen T, Bosch MAAJ, Mali WPTM, Verkooijen HM. Value of preoperative ultrasound-guided axillary lymph node biopsy for preventing completion axillary lymph node dissection in breast cancer: A systematic review and meta-analysis. Ann Surg Oncol. 2014;21(1):51-9.

12. Fuster D, Duch J, Paredes P, Velasco M, Muñoz M, Santamaría G, Fontanillas $M$, Pons F. Preoperative staging of large primary breast cancer with $\left[{ }^{18} \mathrm{~F}\right]$ fluorodeoxyglucose positron emission tomography/computed tomography compared with conventional imaging procedures. J Clin Oncol. 2008;26(29):4746-51.

13. Mamou J, Coron A, Oelze ML, Saegusa-Beecroft E, Hata M, Lee P, Machi J, Yanagihara E, Laugier P, Feleppa EJ. Three-dimensional high-frequency backscatter and envelope quantification of cancerous human lymph nodes. Ultrasound Med Biol. 2011;37(3):345-57.

14. Luke GP, Myers JN, Emelianov SY, Sokolov KV. Sentinel lymph node biopsy revisited: Ultrasound-guided photoacoustic detection of micrometastases using molecularly targeted plasmonic nanosensors. Cancer Res. 2014;74(19): 5397-408.

15. Luo W, Nguyen FT, Zysk AM, Ralston TS, Brockenbrough J, Marks DL, Oldenburg AL, Boppart SA. Optical biopsy of lymph node morphology using optical coherence tomography. Technol Cancer Res Treat. 2005;4(5):539-48

16. McLaughlin RA, Scolaro L, Robbins P, Hamza S, Saunders C, Sampson DD. Imaging of human lymph nodes using optical coherence tomography: Potential for staging cancer. Cancer Res. 2010;70(7):2579-84.

17. Nguyen FT, Zysk AM, Chaney EJ, Adie SG, Kotynek JG, Oliphant UJ, Bellafiore FJ, Rowland KM, Johnson PA, Boppart SA. Optical coherence tomography: The intraoperative assessment of lymph nodes in breast cancer. IEEE Eng Med Biol Mag. 2010;29(2):63-70.

18. Scolaro L, McLaughlin RA, Klyen BR, Wood BA, Robbins PD, Saunders CM, Jacques SL, Sampson DD. Parametric imaging of the local attenuation coefficient in human axillary lymph nodes assessed using optical coherence tomography. Biomed Opt Express. 2012;3(2):366-79.

19. John R, Adie SG, Chaney EJ, Marjanovic M, Tangella KV, Boppart SA. Three-dimensional optical coherence tomography for optical biopsy of lymph nodes and assessment of metastatic disease. Ann Surg Oncol. 2013;20(11):3685-93.

20. Scolaro L, McLaughlin RA, Kennedy BF, Saunders CM, Sampson DD. A review of optical coherence tomography in breast cancer. Photonics Lasers Med. 2014;3(3):225-40.

21. Grieve K, Mouslim K, Assayag O, Dalimier E, Harms F, Bruhat A, Boccara C, Antoine M. Assessment of sentinel node biopsies with full-field optical coherence tomography. Technol Cancer Res Treat. 2015;15(2):266-74.

22. Nolan RM, Adie SG, Marjanovic M, Chaney EJ, South FA, Monroy GL, Shemonski ND, Erickson-Bhatt SJ, Shelton RL, Bower AJ, et al. Intraoperative optical coherence tomography for assessing human lymph nodes for metastatic cancer. BMC Cancer. 2016;16:144.

23. Miyaji K, Furuse A, Nakajima J, Kohno T, Ohtsuka T, Yagyu K, Oka T, Omata S. The stiffness of lymph nodes containing lung carcinoma metastases. Cancer. 1997;80(10):1920-5.

24. Swartz MA. The physiology of the lymphatic system. Adv Drug Deliv Rev. 2001;50(1-2):3-20

25. Doyley MM, Parker KJ. Elastography: General principles and clinical applications. Ultrasound Clin. 2014;9(1):1-11.

26. Glaser KJ, Manduca A, Ehman RL. Review of MR elastography applications and recent developments. J Magn Reson Imaging. 2012;36(4):757-74.

27. Choi JJ, Kang BJ, Kim SH, Lee JH, Jeong SH, Yim HW, Song BJ, Jung SS. Role of sonographic elastography in the differential diagnosis of axillary lymph nodes in breast cancer. J Ultrasound Med. 2011;30(4):429-36.

28. Evans A, Rauchhaus $P$, Whelehan $P$, Thomson $K$, Purdie CA, Jordan LB, Michie CO, Thompson A, Vinnicombe S. Does shear wave ultrasound independently predict axillary lymph node metastasis in women with invasive breast cancer? Breast Cancer Res Treat. 2014;143(1):153-7.

29. Schmitt JM. OCT elastography: Imaging microscopic deformation and strain of tissue. Opt Express. 1998;3(6):199-211. 
30. Kennedy BF, Kennedy KM, Sampson DD. A review of optical coherence elastography: Fundamentals, techniques and prospects. IEEE J Sel Topics Quantum Electron. 2014;20(2):272-88.

31. Wang S, Larin KV. Optical coherence elastography for tissue characterization: A review. J Biophotonics. 2015;8(4):279-302.

32. Mulligan JA, Untracht GR, Chandrasekaran SN, Brown CN, Adie SG. Emerging approaches for high-resolution imaging of tissue biomechanics with optical coherence elastography. IEEE J Sel Topics Quantum Electron. 2016;22(3):6800520.

33. Kennedy BF, McLaughlin RA, Kennedy KM, Chin L, Curatolo A, Tien A, Latham B, Saunders CM, Sampson DD. Optical coherence micro-elastography: Mechanical-contrast imaging of tissue microstructure. Biomed Opt Express. 2014:5(7):2113-24.

34. Kennedy BF, McLaughlin RA, Kennedy KM, Chin L, Wijesinghe P, Curatolo A, Tien A, Ronald M, Latham B, Saunders CM, et al. Investigation of optical coherence micro-elastography as a method to visualize cancers in human breast tissue. Cancer Res. 2015;75(16):3236-45.

35. Wang RK, Kirkpatrick S, Hinds M. Phase-sensitive optical coherence elastography for mapping tissue microstrains in real time. Appl Phys Lett. 2007;90(16):164105.

36. Deserno TM, editor. Biomedical image processing. Berlin Heidelberg: Springer; 2011.

37. Lopez Jl, Kang I, You W-K, McDonald DM, Weaver VM. In situ force mapping of mammary gland transformation. Integr Biol. 2011;3(9):910-21.

38. Plodinec M, Loparic M, Monnier CA, Obermann EC, Zanetti-Dallenbach R, Oertle P, Hyotyla JT, Aebi U, Bentires-Alj M, Lim RYH, et al. The nanomechanical signature of breast cancer. Nat Nanotechnol. 2012;7(11):757-65.

39. Weaver DL. Pathology evaluation of sentinel lymph nodes in breast cancer: Protocol recommendations and rationale. Mod Pathol. 2010;23:S26-32.

40. Li C, Guan G, Huang Z, Johnstone M, Wang RK. Noncontact all-optical measurement of corneal elasticity. Opt Lett. 2012;37(10):1625-7.

41. Wang S, Lopez III AL, Morikawa Y, Tao G, Li J, Larina IV, Martin JF, Larin KV. Noncontact quantitative biomechanical characterization of cardiac muscle using shear wave imaging optical coherence tomography. Biomed Opt Express. 2014:5(7):1980-92.

42. Nguyen T-M, Song S, Arnal B, Huang Z, O'Donnell M, Wang RK. Visualizing ultrasonically induced shear wave propagation using phase-sensitive optical coherence tomography for dynamic elastography. Opt Lett. 2014;39(4):838-41.

43. Kennedy KM, Chin L, McLaughlin RA, Latham B, Saunders CM, Sampson DD, Kennedy BF. Quantitative micro-elastography: Imaging of tissue elasticity using compression optical coherence elastography. Sci Rep. 2015;5:15538.

44. Dong L, Wijesinghe P, Dantuono JT, Sampson DD, Munro PRT, Kennedy BF, Oberai AA. Quantitative compression optical coherence elastography as an inverse elasticity problem. IEEE J Sel Topics Quantum Electron. 2016:22(3):6802211

45. Curatolo A, Villiger M, Lorenser D, Wijesinghe P, Fritz A, Kennedy BF, Sampson DD. Ultrahigh-resolution optical coherence elastography. Opt Lett. 2016;41(1):21-4.

46. Kennedy BF, Malheiro FG, Chin L, Sampson DD. Three-dimensional optical coherence elastography by phase-sensitive comparison of C-scans. J Biomed Opt. 2014;19(7):076006.

47. Kirk RW, Kennedy BF, Sampson DD, McLaughlin RA. Near video-rate optical coherence elastography by acceleration with a graphics processing unit. J Lightw Technol. 2015;33(16):3481-5.

\section{Submit your next manuscript to BioMed Central and we will help you at every step:}

- We accept pre-submission inquiries

- Our selector tool helps you to find the most relevant journal

- We provide round the clock customer support

- Convenient online submission

- Thorough peer review

- Inclusion in PubMed and all major indexing services

- Maximum visibility for your research

Submit your manuscript at www.biomedcentral.com/submit
C) Biomed Central 